Most authors who have treated short children with androgens have refrained from claiming that an increase in predicted height will be followed by an impoved adult height. Our results demonstrate that an increase in predicted height after androgen therapy definitely does not portend an increase in adult height. In warning against over optimistic interpretation of changes in. predicted height resulting from androgen therapy, Bongiovanni (4) suggested that a rapid advance in bone age might occur once therapy was discontinued, although skeletal maturation was not unduly accelerated during therapy. We were able to study the progress of skeletal maturation of eight boys, two of whom seemed to have such an acceleration, but the others did not and continued to have predicted heights greater than pretreatment predicted height for as long as they were followed.

Our results have an additional implication for the evaluation of therapies designed to affect adult height in children with severely delayed bone ages. Because $50 \%$ of such boys fail to attain at least their predicted height less $5.1 \mathrm{~cm}$, a treatment having no effect on predicted height but causing all treated children to reach their predicted height as adults would represent a significant improvement in outcome for these children. Thus, follow-up until adult height is attained is required before a therapy can be deemed effective or ineffective.

\section{REFERENCES AND NOTES}

1. Bayer L. M. and Bayley N.: Growth pattern shifts in healthy children: spontaneous and induced. J. Pediatr., 62: 631 (1963).
2. Bayley N. and Pinneau S. R.: Tables for predicting adult height from skeletal age: revised for use with the Greulich-Pyle hand standards. J. Pediatr., 40: 423 (1952).

3. Bettmann H. K., Goldman H. S., Abramowicz M., and Sobel E. H.: Oxandrolone treatment of short stature: effect on predicted mature height. J. Pediatr. 79: 1018 (1971).

4. Bongiovanni A. M.: Commentary. Anabolic drugs to promote growth. Pediatrics, 27: 519 (1961).

5. Greulich W. W. and Pyle S. I.: Radiographic atlas of skeletal development of the hand and wrist. 2nd ed., (Stanford University Press, Stanford, 1959).

6. Kaplan J. G., Moshang T. Jr., Bernstein R., Parks, J. S., and Bongiovanni, A M.: Constitutional delay of growth and development: effects of treatment with androgens. J Pediatr. 82: 38 (1973).

7. Reilly W. A and Gordon G. S.: Dissociation of growth-stimulating and skeleton maturing actions of the synthetic androgen, fluoxymesterone. J. Pediatr., 59: 188 (1961).

8. Rosenfeld R. G., Northcraft G. B., and Hintz R. L.: A prospective, randomized study of testosterone treatment of constitutional delay of growth and development in male adolescents. Pediatrics, 69: 681 (1982).

9. Swinscow T. D. V.: Statistics at square one. 7 th ed. (British Medical Association, London, 1980).

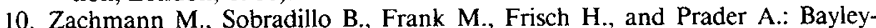
Pinneau, Roche-Wainer-Thissen, and Tanner height predictions in normal children and in patients with various pathological conditions. J. Pediatr., 93 749 (1978).

11. Presented to the Society for Pediatric Research, Washington, D.C., May 3. 1983.

12. Requests for reprints should be addressed to: Dr. S. L. Blethen, P.O. Box 14871, St. Louis, MO, 63178.

13. This research was supported in part by grants RR-36 from the Division of Research Resources and AMO-5105 from the NIADDK of the National Institutes of Health.

14. Received for publication April 19, 1983.

15. Accepted for publication August 9,1983

\title{
Cold Air Challenge of Airway Hyperreactivity in Children: Practical Application and Theoretical Aspects $^{(46)}$
}

\author{
M. $\mathrm{ZACH}^{(48)}$ G. POLGAR, ${ }^{(47)}$ H. KUMP, AND P. KROISEL \\ Department of Pediatrics, University of Graz, Graz, Austria and Children's Hospital of Michigan, Wayne State \\ University, Detroit, Michigan, USA
}

\section{Summary}

In 23 children with asthma and 18 healthy controls, cold air challenge $(\mathrm{CACh})$ was done twice during the same half day, and in the asthmatics a third time together with a histamine challenge (HCh) 2 wk later. Pulmonary functions were tested before and after each challenge. No overlapping of individual responses to $\mathrm{CACh}$ in seven forced expiratory flow tests proved the power of discrimination of this technique in children. The limits of "normal" reactions ranged from minus $9 \%$ for larger airway-related to $26 \%$ for smaller airway-related flows. Short-term reproducibility of induced changes, in percentage of baseline, was excellent $(r=0.815-0.954)$; in percentage of predicted postchallenge abnormality it was even bettern $(r=0.926-0.975)$. The response in small airway-related flow rates $(-43.1 \pm 12.8$ to $-51.9 \pm$ $16.8 \%$ of baseline) was much larger than in others $(-27.6 \pm 14.6$ to $-32.1 \pm 17.3 \%$ of baseline). This, the different baseline-toresponse correlations in various measurements, and the divergent dose response to colder versus less cold air in large $(60.7 \pm 21.9$ versus $65.4 \pm 21.5 \%$ predicted, postchallenge values) and small airway-related tests $(28.9 \pm 18.7$ versus $29.5 \pm 15.1 \%$ predicted, postchallenge values) in asthmatic children suggest a predetermined, small airway-related limitation of individual reactivity, which is independent of the baseline situation. All asthmatics responded positively to $\mathrm{HCh}$ but quantitative results of the two methods did not correlate. Responses to $\mathrm{CACh}$ also better characterized the clinical severity of asthma than those to $\mathrm{HCh}$. Determining the individual optimum by a bronchodilator and the physiologic abnormality by $\mathrm{CACh}$, the whole functional dimension of a child's asthma can be established.

\section{Abbreviations}

CACh, cold air challenge

FEF $_{25-75}$, forced expiratory flow during mid-half of FVC

$\mathrm{FEV}_{1}$, forced expiratory volume in one second

FRC, functional residual capacity 
FVC, forced (expiratory) vital capacity

$\mathrm{HCh}$, histamine inhalation challenge

Mid-VC ratio, $\frac{\dot{\mathrm{V}}_{\text {Emax50\%VC-MEFV }}}{\dot{\mathrm{V}}_{\text {Imax50\%VC-MIFV }}}$

MEFV, maximum expiratory flow-volume curve

MIFV, maximum inspiratory flow-volume curve

MVV, maximum voluntary ventilation

$\mathrm{PC}_{20}$-histamine, provocative concentration of histamine causing a $20 \%$ or greater fall of $\mathbf{F E V}$.

PEF, peak expiratory flow

PEFV, partial expiratory flow-volume curve

PFT, pulmonary function tests (or testing)

$R V$, residual volume

TLC, total lung capacity

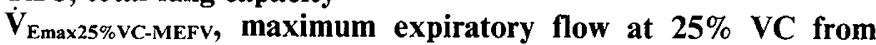
MEFV

$\dot{\mathrm{V}}_{\text {Emax50\%VC-MEFv, maximum expiratory flow at } 50 \% \mathrm{VC} \text { from }}$ MEFV

$\dot{\mathrm{V}}_{\text {Emax60\%TLC-MEFv, maximum expiratory flow at } 60 \% \text { TLC, from }}$ MEFV

$\dot{\mathrm{V}}_{\text {Emax25\%VC-PEFv, maximum expiratory flow at } 25 \% \mathrm{VC} \text { from }}$ PEFV

$\dot{\mathrm{V}}_{\text {Emax-isovol.60\% TLC-MEFv, maximum expiratory flow at } 60 \% \text { of base- }}$ line TLC, from MEFV

$\dot{\mathrm{V}}_{\text {Imax50\%VC-MIFV, maximum inspiratory flow at } 50 \% \mathrm{VC} \text { from }}$ MIFV

VC, vital capacity (inspiratory)

Bronchial hyperreactivity has been recognized as a significant factor in causing the clinical symptoms of the asthmatic syndrome (1). For testing this phenomenon by non-specific challenges, standardized methods have been developed. These include the use of pharmacologically active agents and exercise (8, $11,12,36$ ).

The mechanism by which exercise causes bronchoconstriction was closely linked to the effect of airway cooling produced by an increased heat exchange during voluntary hyperventilation with subfreezing air (39, 40, 13-16). Although the final pathways for exercise and $\mathrm{CACh}$ are not fully understood, reliable separation of adult patients with asthma from healthy controls was possible by CACh (17). Because the asthmatic syndrome in children and adults is basically similar and because of the growing evidence of different pediatric respiratory diseases being followed by bronchial hyperreactivity, the need exists for a conclusive study of $\mathrm{CACh}$ in children.

The investigation reported here was aimed at: $l$ ) establishing the feasibility of $\mathrm{CACh}$ for testing bronchial hyperreactivity in children, 2) examining the discriminative power of the test between children with the asthmatic syndrome and healthy controls, and 3) testing the reproducibility and time course of cold air-induced reactions. The usefulness of this test in studying the nature as well as the limits of hyperreactivity was also explored.

\section{MATERIALS AND METHODS}

A group of 23 children with asthma (group A) and a group of 18 healthy control (group HC) children participated in the study. Group A consisted of 14 boys and 9 girls (mean age, 117/12 yr; range, $79 / 12$ to $153 / 12$ ). Fifty children had been randomly selected from 250 attending the outpatient asthma clinic. Of these, 27 were excluded for the following reasons: 12 were too young for pulmonary function testing, 5 had seasonal symptoms, 4 resided too far from the hospital, 2 were unwilling to participate, and 4 required regular systemic corticosteroid therapy. All 23 patients in Group A met the clinical definition of the asthma syndrome (1), having had repeated episodes of reversible intrathoracic airway obstruction with typical findings by PFT. They required medication with various drugs under regular supervision; however, none received systemic corticosteroid treatment. Fourteen of 20 patients tested had two or more positive skin reactions to common allergens. Nineteen children had a history of exercise induced bronchospasm, seven of them responded more severely to outdoor exercise in the winter.

Group HC consisted of 12 boys and six girls (mean age, $11 \% / 12$ yr; range, $65 / 12$ to $148 / 12$ ) carefully evaluated by a uniform questionnaire for negative personal and family history of respiratory and allergic disorders. They never smoked and had no respiratory tract infections for at least $6 \mathrm{wk}$ before the study. Informed consents were obtained from parents and children of both groups.

Patients in Group A were requested to discontinue treatment with sodium chromoglycate and inhaled corticosteroids 3 wk prior, with oral theophyllines $3 \mathrm{~d}$ prior, and with inhaled sympathomimetics $24 \mathrm{~h}$ before the investigation. By chance, none of the patients had sufficiently bothersome symptoms during the off-medication period to indicate their exclusion from the study.

$C A C h$ was given by a home built equipment similar to the one described by Simonssen et al. (37). Two rectangular polyethelene tanks, one inside the other, insulated with styrofoam and with overall dimensions of $75 \times 50 \mathrm{~cm}$, served as a container for a dry ice-in-acetone bath. A 5-cm diameter, 1-mm thick, $140-\mathrm{cm}$ long coiled copper tube was inserted through holes in the lid of the inner container and submerged in the bath when the lid was closed. The outlet was equipped with a two-way valve, a thermistor probe and the mouthpiece. On the expiratory side of the valve the sampler from a $\mathrm{CO}_{2}$ analyzer (Jaeger $\mathrm{CO}_{2}$-Test) provided for continuous monitoring of the expired air, and a ventilometer (Draeger) measured minute ventilation. Through the inlet side of the copper tube enough $\mathrm{CO}_{2}$ was continuously added during the test to keep the patient eucapnic. With this arrangement, inhaled air temperatures of -10 to $-20^{\circ} \mathrm{C}$ could be maintained. During cold air inhalation for $4 \mathrm{~min}$, the subjects were continuously coached to hyperventilate at $75 \%$ of their MVV level, as calculated from $\mathrm{FEV}_{1}$ and monitored by the ventilometer.

Instead of dry air from a tank, room air was used for hyperventilation because the children objected to the "taste" of the compressed air, and because when the air with an average room temperature of $+20 \pm 2^{\circ} \mathrm{C}$ and $60 \pm 10 \%$ relative humidity was cooled to around $-15^{\circ} \mathrm{C}$, the very low absolute water content imposed an almost equal evaporation-related thermal burden on the respiratory mucosa as would have dry air.

PFT's were done for baseline (prechallenge) determinations and for measuring the responses to challenges on a water-filled spirometer (Jaeger Spiro-Junior), by a helium dilution method on a direct read-out instrument (Jaeger FRC-Test), and on a pneumotachograph spirometer and X-Y recorder (Jaeger Pneumotest Junior). Recording and evaluation were done in accordance with standardized guidelines (42). The children performed all manoeuvres in the sitting position wearing a nose clip. The functions measured or calculated were: VC, FRC, RV, TLC, FVC, FEV $1, F E_{25-75}$, PEF, $\dot{\mathrm{V}}_{\text {Emax } 50 \% \mathrm{VC}-\mathrm{MEFV}}, \dot{\mathrm{V}}_{\text {Emax60\%TLC-MEFV, }}$ $\dot{\mathrm{V}}_{\text {Emax-isovol.60\%TLC-MEFV }}, \dot{\mathrm{V}}_{\text {Emax } 25 \% \mathrm{VC}-\mathrm{MEFV}}, \dot{\mathrm{V}}_{\text {Imax } 50 \% \mathrm{VC}-\mathrm{MIFV}}$, midVC-

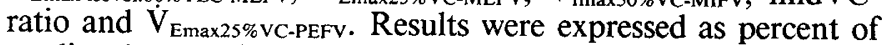
predicted normals based on accepted reference standards (34, $38,45)$.

Histamine inhalation challenge was given with $\mathrm{CACh}$ by a standardized procedure (8). Histamine acid phosphate aersol, produced by a jet type nebulizer (Wright), was inhaled in up to nine dosage steps $(0.03-10.0 \mathrm{mg} / \mathrm{ml})$ at 3-min intervals. FEV was measured $2.5 \mathrm{~min}$ after each inhalation. The series was complete at the concentration which caused a $20 \%$ or greater fall in $\mathrm{FEV}_{1}$. The $\mathrm{PC}_{20 \text {-histamine value was obtained by linear interpo- }}$ lation between the last two points of the log dose-response curve $(11,35)$. PFTs before and 3-5 min after the last inhalation were measured as for CACh. Recovery was recorded by spirometry in 5 -min intervals for $30 \mathrm{~min}$ or until $\mathrm{FEV}_{1}$ reached at least $95 \%$ of the pre-HCh value. 
The sequence of testing comprised the following 13 steps: 1) The subjects of both groups had a 30-min rest in the laboratory $\left(20^{\circ} \mathrm{C}, 60 \%\right.$ relative humidity) between $8: 00 \mathrm{AM}$ and 9:00 AM. 2) The children were trained in PFT manoeuvres until performing with maximal effort as judged by reproducibility. 3) Baseline PFTs were performed in triplicate in the following order: FRC, VC, FVC, and after a 5-min break, PEFV and MIFV-MEFV. 4) CACh was given. 5) The children were asked about their subjective feeling of "chest tightness" as a result of CACh. 6) Five minutes after the completion of the challenge, PEFV, MIFVMEFV, FVC, FRC, and VC were recorded. 7) Fifteen minutes after $\mathrm{CACh}$ the forced spirometric tests were repeated. 8) After a rest period of $2 \mathrm{~h}$, the CACh procedure and the PFTs were performed for a second time. If children had required bronchodilator medication after $\mathrm{CACh} \mathrm{I}$, this interval was prolonged to 4 h. 9) Thirty minutes after the second CACh, $\mathrm{FEV}_{1}$ was again measured in the children of Group A. If it was less than $100 \%$ of baseline or $80 \%$ of predicted value, bronchodilator medication was given. Salbutamol (Albuterol) $(0.2 \mathrm{ml}$ of the $0.5 \%$ solution per $\mathrm{kg}$ body weight, diluted to $2 \mathrm{ml}$ ) from a jet-type nebulizer (Pari-Inhalierboy 360 Nebulizer) was inhaled through a mouthpiece by slow tidal breathing. Fifteen minutes later, forced spirometric tests were performed again. 10) The results of each CACh were expressed in two ways: as actual PFT values after the challenge (in percentage of predicted) and as PFT changes induced by the challenge (in percentage of baseline).

In the following parts of the protocol, only children of Group A participated: 11) During the week after the first two CAChs, the children measured and recorded their PEF twice a day on a Wright peak flow meter. The results were summarized as a mean and coefficient of variation for each child. 12) On $1 \mathrm{~d}, 2$ wk after the first tests, a third $\mathrm{CACh}$ and a $\mathrm{HCh}$ were performed in random order after baseline PFTs as under step 3. Upon the completion of these challenges, Salbutamol was administered as under step 9. 13) After this day of testing, PEF was again measured for $1 \mathrm{wk}$ as under step 11.

The statistical evaluation of all results was performed using the $t$ tests for paired and unpaired samples and regression analyses by the least squares method. A $P$ value $\leq 0.05$ indicated a significant difference or correlation.

\section{RESULTS}

Altogether $52 \mathrm{CAChs}$ and $18 \mathrm{HChs}$ were done in Group A and 33 CAChs in Group HC. Five children with asthma appeared at one or another testing without a preceding break in medication; these tests are not included in the above total. Others, including three of Group $\mathrm{HC}$, were absent from one or more testings. Different total numbers of patients in different categories of Tables 1-6 resulted from these exclusions and absences.

Two children in Group A had to be treated with a bronchodilator aerosol after CACh. One had unremitting discomfort associated with bronchoconstriction, the other could perform only one FEV-manoeuvre before requiring a bronchodilator treatment. This was the only one with an incomplete assessment protocol.

Baseline PFT results before each CACh are shown in Table 1. The baseline values before three cold air challenges were comparable in each group; however, children in Group A demonstrated considerable intraindividual variation.

Cold air-induced changes in percentage of baseline values for pulmonary functions $5 \mathrm{~min}$ after all three challenges are shown in Table 2. There was no overlap of individual changes between Groups $\mathrm{A}$ and $\mathrm{HC}$ in seven forced expiratory flow tests $\left(\mathrm{FEV}_{1}\right.$,

Table 1. Baseline values*

\begin{tabular}{|c|c|c|c|c|c|}
\hline & \multicolumn{2}{|c|}{ CACh I } & \multicolumn{2}{|c|}{ CACh II } & \multirow{2}{*}{$\begin{array}{c}\text { CACh III } \\
\begin{array}{c}\mathrm{A} \\
(n=13)\end{array}\end{array}$} \\
\hline & $\begin{array}{c}\mathrm{A} \\
(n=23)\end{array}$ & $\begin{array}{c}\mathrm{HC} \\
(n=18)\end{array}$ & $\begin{array}{c}\mathrm{A} \\
(n=16)\end{array}$ & $\begin{array}{c}\mathrm{HC} \\
(n=15)\end{array}$ & \\
\hline $\begin{array}{l}\text { FVC } \\
\text { (\% predicted) }\end{array}$ & $\begin{array}{c}100.4 \pm 12.6 \\
(76-122)\end{array}$ & $\begin{array}{r}103.1 \pm 8.8 \\
(86-125)\end{array}$ & $\begin{array}{c}102.6 \pm 12.1 \\
(76-124)\end{array}$ & $\begin{array}{c}103.5 \pm 10.8 \\
(84-128)\end{array}$ & $\begin{array}{c}93.8 \pm 17.2 \\
(53-121)\end{array}$ \\
\hline $\begin{array}{l}\text { FEV } \\
(\% \text { predicted })\end{array}$ & $\begin{array}{c}85.8 \pm 17.5 \\
(40-123)\end{array}$ & $\begin{array}{r}97.7 \pm 8.8 \\
(83-114)\end{array}$ & $\begin{array}{c}87.2 \pm 17.1 \\
(64-123)\end{array}$ & $\begin{array}{r}95.7 \pm 8.8 \\
(82-113)\end{array}$ & $\begin{array}{c}80.5 \pm 19.8 \\
(47-123)\end{array}$ \\
\hline $\begin{array}{l}\mathrm{FEV}_{1 / \mathrm{FvC}} \\
(\%)\end{array}$ & $\begin{array}{c}78.6 \pm 11.0 \\
(46-98)\end{array}$ & $\begin{array}{c}87.9 \pm 4.3 \\
(81-95)\end{array}$ & $\begin{array}{c}78.2 \pm 9.7 \\
(58-98)\end{array}$ & $\begin{array}{c}85.7 \pm 4.6 \\
(78-92)\end{array}$ & $\begin{array}{c}77.2 \pm 13.2 \\
(51-98)\end{array}$ \\
\hline $\begin{array}{l}\mathrm{FEF}_{25-75} \\
\text { (\% predicted) }\end{array}$ & $\begin{array}{c}72.5 \pm 28.4 \\
(18-123)\end{array}$ & $\begin{array}{c}95.6 \pm 14.3 \\
(72-122)\end{array}$ & $\begin{array}{c}67.9 \pm 24.5 \\
(30-123)\end{array}$ & $\begin{array}{c}92.8 \pm 17.2 \\
(60-119)\end{array}$ & $\begin{array}{c}64.0 \pm 30.5 \\
(24-123)\end{array}$ \\
\hline (\% predicted) & $\begin{array}{c}96.1 \pm 16.2 \\
(52-120)\end{array}$ & $\begin{array}{c}100.7 \pm 10.4 \\
(87-124)\end{array}$ & $\begin{array}{l}99.0 \pm 10.0 \\
(79-118)\end{array}$ & $\begin{array}{c}102.8 \pm 12.4 \\
(83-129)\end{array}$ & $\begin{array}{c}94.1 \pm 16.0 \\
(52-117)\end{array}$ \\
\hline $\begin{array}{l}\text { FRC } \\
\quad(\% \text { predicted })\end{array}$ & $\begin{array}{c}103.3 \pm 18.0 \\
(67-146)\end{array}$ & $\begin{array}{c}97.4 \pm 15.5 \\
(77-130)\end{array}$ & $\begin{array}{c}95.8 \pm 18.1 \\
(71-139)\end{array}$ & $\begin{array}{c}96.1 \pm 24.0 \\
(73-162)\end{array}$ & $\begin{array}{c}101.8 \pm 17.6 \\
(78-131)\end{array}$ \\
\hline $\begin{array}{l}\text { TLC } \\
\text { (\% predicted) }\end{array}$ & $\begin{array}{c}95.7 \pm 11.6 \\
(78-118)\end{array}$ & $\begin{array}{c}94.7 \pm 10.1 \\
(80-112)\end{array}$ & $\begin{array}{r}97.3 \pm 9.8 \\
(77-111)\end{array}$ & $\begin{array}{r}95.2 \pm 9.1 \\
(81-112)\end{array}$ & $\begin{array}{c}92.8 \pm 15.4 \\
(62-120)\end{array}$ \\
\hline $\begin{array}{l}\text { RV } \\
\text { (\% predicted) }\end{array}$ & $\begin{array}{c}92.7 \pm 37.6 \\
(42-192)\end{array}$ & $\begin{array}{c}71.9 \pm 19.5 \\
(55-119)\end{array}$ & $\begin{array}{c}84.3 \pm 21.4 \\
(59-121)\end{array}$ & $\begin{array}{c}63.7 \pm 25.2 \\
(42-140)\end{array}$ & $\begin{array}{c}81.0 \pm 21.8 \\
(47-125)\end{array}$ \\
\hline $\begin{array}{l}\mathrm{RV} / \text { /Ruc } \\
(\%)\end{array}$ & $\begin{array}{c}20.7 \pm 7.9 \\
(12-40)\end{array}$ & $\begin{array}{c}15.9 \pm 4.0 \\
(11-26)\end{array}$ & $\begin{array}{c}18.5 \pm 4.3 \\
(13-26)\end{array}$ & $\begin{array}{c}15.3 \pm 4.9 \\
(11-30)\end{array}$ & $\begin{array}{c}19.9 \pm 5.4 \\
(13-34)\end{array}$ \\
\hline $\begin{array}{l}\text { PEF } \\
\text { (\% predicted) }\end{array}$ & $\begin{array}{c}92.7 \pm 12.7 \\
(73-120)\end{array}$ & $\begin{array}{c}98.5 \pm 13.8 \\
(70-121)\end{array}$ & $\begin{array}{c}90.5 \pm 15.2 \\
(75-128)\end{array}$ & $\begin{array}{r}95.4 \pm 9.7 \\
(80-113)\end{array}$ & $\begin{array}{c}84.0 \pm 24.1 \\
(54-130)\end{array}$ \\
\hline $\begin{array}{l}\dot{\mathrm{V}}_{\text {Emax } 50 \% \mathrm{VC}-\mathrm{MEFV}} \\
(\% \text { predicted })\end{array}$ & $\begin{array}{c}67.2 \pm 24.5 \\
(20-115)\end{array}$ & $\begin{array}{c}87.8 \pm 16.4 \\
(54-113)\end{array}$ & $\begin{array}{c}60.9 \pm 19.2 \\
(31-107)\end{array}$ & $\begin{array}{c}79.0 \pm 15.7 \\
(52-99)\end{array}$ & $\begin{array}{c}52.5 \pm 27.0 \\
(21-107)\end{array}$ \\
\hline $\begin{array}{r}\dot{\mathrm{V}}_{\text {Emax60\%TLC-MEFV }} \\
\text { (\% predicted) }\end{array}$ & $\begin{array}{c}68.0 \pm 27.7 \\
(16-125)\end{array}$ & $\begin{array}{c}92.2 \pm 19.8 \\
(56-120)\end{array}$ & $\begin{array}{c}62.1 \pm 22.8 \\
(29-115)\end{array}$ & $\begin{array}{c}84.9 \pm 16.2 \\
(58-108)\end{array}$ & $\begin{array}{c}53.6 \pm 30.2 \\
(19-108)\end{array}$ \\
\hline $\begin{array}{c}\dot{\mathrm{V}}_{\text {Emax } 25 \% \mathrm{VC-MEFV}} \\
\text { (\% predicted) }\end{array}$ & $\begin{array}{c}53.8 \pm 23.6 \\
(15-103)\end{array}$ & $\begin{array}{c}84.4 \pm 16.3 \\
(55-114)\end{array}$ & $\begin{array}{c}48.8 \pm 19.5 \\
(22-95)\end{array}$ & $\begin{array}{c}77.6 \pm 16.9 \\
(50-98)\end{array}$ & $\begin{array}{c}45.9 \pm 29.9 \\
(19-107)\end{array}$ \\
\hline $\begin{array}{l}\text { Mid VC-ratio } \\
(\%)\end{array}$ & $\begin{array}{c}74.6 \pm 27.9 \\
(27-128)\end{array}$ & $\begin{array}{c}98.8 \pm 34.7 \\
(45-183)\end{array}$ & $\begin{array}{c}63.7 \pm 27.3 \\
(39-133)\end{array}$ & $\begin{array}{c}91.8 \pm 38.9 \\
(45-195)\end{array}$ & $\begin{array}{c}55.7 \pm 28.2 \\
(25-114)\end{array}$ \\
\hline $\begin{array}{c}\dot{\mathrm{V}}_{\text {Emax } 25 \% \mathrm{VC}-\mathrm{PEFV}} \\
\text { (\% predicted) }\end{array}$ & $\begin{array}{c}52.5 \pm 22.2 \\
(17-93) \\
\end{array}$ & $\begin{array}{c}73.8 \pm 17.3 \\
(52-105) \\
\end{array}$ & $\begin{array}{c}49.8 \pm 18.7 \\
(25-91)\end{array}$ & $\begin{array}{c}69.0 \pm 14.1 \\
(50-91)\end{array}$ & $\begin{array}{c}43.5 \pm 24.7 \\
(21-97)\end{array}$ \\
\hline
\end{tabular}

*Values are mean \pm SD (range). Abbreviations, see "Abbreviations." 
Table 2. Cold air-induced changes* (5 min after CACh)

\begin{tabular}{|c|c|c|c|c|c|}
\hline & \multicolumn{2}{|c|}{ CACh I } & \multicolumn{2}{|c|}{ CACh II } & \multirow{2}{*}{$\begin{array}{c}\text { CACh III } \\
\begin{array}{c}\mathrm{A} \\
(n=13)\end{array}\end{array}$} \\
\hline & $\begin{array}{c}\mathrm{A} \\
(n=22) \dagger\end{array}$ & $\begin{array}{c}\mathrm{HC} \\
(n=18)\end{array}$ & $\begin{array}{c}\mathrm{A} \\
(n=16)\end{array}$ & $\begin{array}{c}\mathrm{HC} \\
(n=15)\end{array}$ & \\
\hline $\begin{array}{l}\Delta \mathrm{FVC} \\
\quad(\% \text { baseline })\end{array}$ & $\begin{array}{r}-14.4 \pm 17.6 \\
(+4 \text { to }-72)\end{array}$ & $\begin{array}{l}-1.4 \pm 3.6 \\
(+3 \text { to }-11)\end{array}$ & $\begin{array}{r}-12.3 \pm 10.9 \\
(0 \text { to }-42)\end{array}$ & $\begin{array}{c}-0.8 \pm 2.6 \\
(+4 \text { to }-7)\end{array}$ & $\begin{array}{r}-13.2 \pm 13.7 \\
(+3 \text { to }-38)\end{array}$ \\
\hline $\begin{array}{l}\triangle F E V_{1} \\
\text { (\% baseline) }\end{array}$ & $\begin{array}{r}-31.2 \pm 15.4 \\
(-12 \text { to }-65)\end{array}$ & $\begin{array}{r}-2.4 \pm 3.1 \\
(+4 \text { to }-7)\end{array}$ & $\begin{array}{r}-27.6 \pm 14.6 \\
(-12 \text { to }-62)\end{array}$ & $\begin{array}{c}-1.9 \pm 2.3 \\
(+3 \text { to }-5)\end{array}$ & $\begin{array}{r}-32.1 \pm 17.3 \\
(-11 \text { to }-63)\end{array}$ \\
\hline $\begin{array}{l}\Delta \mathrm{FEV}_{25-75} \\
\quad(\% \text { baseline })\end{array}$ & $\begin{array}{r}-52.9 \pm 15.7 \\
(-20 \text { to }-73)\end{array}$ & $\begin{array}{l}-3.3 \pm 8.8 \\
(+19 \text { to }-18)\end{array}$ & $\begin{array}{r}-46.9 \pm 15.1 \\
(-21 \text { to }-65)\end{array}$ & $\begin{array}{l}-6.6 \pm 4.7 \\
(+6 \text { to }-12)\end{array}$ & $\begin{array}{r}-50.8 \pm 17.3 \\
(-28 \text { to }-79)\end{array}$ \\
\hline $\begin{array}{l}\Delta \mathrm{VC} \\
\quad(\% \text { baseline })\end{array}$ & $\begin{array}{r}-8.8 \pm 10.6 \\
(+4 \text { to }-38)\end{array}$ & $\begin{array}{l}+0.9 \pm 4.1 \\
(+12 \text { to }-6)\end{array}$ & $\begin{array}{c}-3.3 \pm 7.7 \\
(+7 \text { to }-18)\end{array}$ & $\begin{array}{l}+1.0 \pm 5.7 \\
(+19 \text { to }-8)\end{array}$ & $\begin{array}{l}-6.9 \pm 9.8 \\
(+5 \text { to }-31)\end{array}$ \\
\hline $\begin{array}{l}\Delta \mathrm{FRC} \\
\quad(\% \text { baseline })\end{array}$ & $\begin{array}{c}+12.4 \pm 13.3 \\
(+33 \text { to }-16)\end{array}$ & $\begin{array}{l}+0.8 \pm 9.1 \\
(+17 \text { to }-17)\end{array}$ & $\begin{array}{c}+13.5 \pm 12.3 \\
(+36 \text { to }-5)\end{array}$ & $\begin{array}{l}-5.5 \pm 10.2 \\
(+18 \text { to }-22)\end{array}$ & $\begin{array}{c}+14.2 \pm 9.3 \\
(+28 \text { to }-4)\end{array}$ \\
\hline $\begin{array}{l}\Delta \mathrm{TLC} \\
\quad \text { (\% baseline })\end{array}$ & $\begin{array}{l}+5.4 \pm 7.6 \\
(+24 \text { to }-7)\end{array}$ & $\begin{array}{l}+0.2 \pm 5.2 \\
(+9 \text { to }-11)\end{array}$ & $\begin{array}{l}+5.1 \pm 5.0 \\
(+13 \text { to }-7)\end{array}$ & $\begin{array}{c}+1.8 \pm 3.2 \\
(+7 \text { to }-5)\end{array}$ & $\begin{array}{l}+4.2 \pm 6.3 \\
(+14 \text { to }-11)\end{array}$ \\
\hline $\begin{array}{l}\Delta \mathrm{RV} \\
\quad(\% \text { baseline })\end{array}$ & $\begin{array}{l}+60.4 \pm 37.5 \\
(+150 \text { to }+2)\end{array}$ & $\begin{array}{l}+0.2 \pm 34.4 \\
(+77 \text { to }-56)\end{array}$ & $\begin{array}{r}+45.1 \pm 18.9 \\
(+80 \text { to }+16)\end{array}$ & $\begin{array}{r}+13.3 \pm 32.5 \\
(+77 \text { to }-21)\end{array}$ & $\begin{array}{c}+53.4 \pm 28.7 \\
(+98 \text { to }+9)\end{array}$ \\
\hline $\begin{array}{l}\triangle \mathrm{PEF} \\
\quad(\% \text { baseline })\end{array}$ & $\begin{array}{r}-27.8 \pm 18.4 \\
(-2 \text { to }-57)\end{array}$ & $\begin{array}{l}-0.9 \pm 5.8 \\
(+13 \text { to }-11)\end{array}$ & $\begin{array}{r}-17.1 \pm 13.9 \\
(-1 \text { to }-42)\end{array}$ & $\begin{array}{c}-0.6 \pm 4.9 \\
(+9 \text { to }-10)\end{array}$ & $\begin{array}{r}-23.6 \pm 14.7 \\
(-3 \text { to }-56)\end{array}$ \\
\hline $\begin{array}{c}\Delta \dot{\mathrm{V}}_{\text {Emax } 50 \% \mathrm{VC}-\mathrm{MEFV}} \\
(\% \text { baseline })\end{array}$ & $\begin{array}{c}-50.8 \pm 18.9 \\
(-23 \text { to }-86)\end{array}$ & $\begin{array}{l}-7.3 \pm 9.5 \\
(+13 \text { to }-23)\end{array}$ & $\begin{array}{r}-39.1 \pm 13.4 \\
(-26 \text { to }-68)\end{array}$ & $\begin{array}{l}-5.4 \pm 8.3 \\
(+13 \text { to }-18)\end{array}$ & $\begin{array}{r}-44.4 \pm 16.2 \\
(-24 \text { to }-77)\end{array}$ \\
\hline $\begin{array}{l}\Delta \dot{\mathrm{V}}_{\text {Emax60\%TLC-MEFV }} \\
\text { (\% baseline) }\end{array}$ & $\begin{array}{c}-60.7 \pm 19.8 \\
(-29 \text { to }-88)\end{array}$ & $\begin{array}{l}-6.8 \pm 12.3 \\
(+18 \text { to }-26)\end{array}$ & $\begin{array}{r}-44.8 \pm 16.4 \\
(-26 \text { to }-75)\end{array}$ & $\begin{array}{l}-4.7 \pm 10.5 \\
(+21 \text { to }-19)\end{array}$ & $\begin{array}{c}-46.9 \pm 16.2 \\
(-26 \text { to }-75)\end{array}$ \\
\hline $\begin{array}{l}\Delta \mathrm{V}_{\text {Emax-isovol.60\%TLC-MEFV }} \\
(\% \text { baseline })\end{array}$ & $\begin{array}{c}-59.8 \pm 16.0 \\
(-30 \text { to }-81)\end{array}$ & $\begin{array}{l}-2.5 \pm 17.3 \\
(+38 \text { to }-28)\end{array}$ & $\begin{array}{c}-49.4 \pm 15.8 \\
(-30 \text { to }-82)\end{array}$ & $\begin{array}{l}-6.0 \pm 13.1 \\
(+21 \text { to }-28)\end{array}$ & $\begin{array}{c}-55.6 \pm 13.5 \\
(-36 \text { to }-85)\end{array}$ \\
\hline $\begin{array}{c}\Delta \dot{\mathrm{V}}_{\text {Emax25\%VC-MEFV }} \\
(\% \text { baseline })\end{array}$ & $\begin{array}{r}-51.7 \pm 18.3 \\
(-26 \text { to }-87)\end{array}$ & $\begin{array}{l}-8.8 \pm 8.2 \\
(+6 \text { to }-21)\end{array}$ & $\begin{array}{c}-43.1 \pm 12.8 \\
(-29 \text { to }-72)\end{array}$ & $\begin{array}{l}-7.5 \pm 6.5 \\
(+4 \text { to }-19)\end{array}$ & $\begin{array}{r}-46.8 \pm 12.0 \\
(-29 \text { to }-73)\end{array}$ \\
\hline $\begin{array}{c}\Delta \dot{\mathrm{V}}_{\text {lmax } 50 \% \mathrm{VC}-\mathrm{MIFV}} \\
(\% \text { baseline })\end{array}$ & $\begin{array}{r}-13.5 \pm 21.0 \\
(+21 \text { to }-56)\end{array}$ & $\begin{array}{l}+5.6 \pm 13.2 \\
(+35 \text { to }-11)\end{array}$ & $\begin{array}{l}-6.1 \pm 14.1 \\
(+30 \text { to }-28)\end{array}$ & $\begin{array}{l}+4.2 \pm 16.0 \\
(+33 \text { to }-23)\end{array}$ & $\begin{array}{r}-11.1 \pm 16.6 \\
(+8 \text { to }-50)\end{array}$ \\
\hline $\begin{array}{c}\Delta \dot{\mathrm{V}}_{\text {Emax } 25 \% \mathrm{VC}-\mathrm{PEFV}} \\
(\% \text { baseline }) \\
\end{array}$ & $\begin{array}{r}-51.9 \pm 16.8 \\
(-27 \text { to }-80)\end{array}$ & $\begin{array}{l}-8.2 \pm 8.7 \\
(+8 \text { to }-19)\end{array}$ & $\begin{array}{c}-45.3 \pm 16.9 \\
(-27 \text { to }-85)\end{array}$ & $\begin{array}{l}-8.1 \pm 6.9 \\
(+6 \text { to }-18)\end{array}$ & $\begin{array}{r}-50.1 \pm 14.8 \\
(-26 \text { to }-73)\end{array}$ \\
\hline
\end{tabular}

* Values are mean \pm SD (range). Abbreviations, see "Abbreviations."

$\dagger n=23$ for $\mathrm{FVC}, \mathrm{FEV}_{1}$, and $\mathrm{FEF}_{25-75}$.

$\mathrm{FEF}_{25-75}, \dot{\mathrm{V}}_{\text {Emax } 50 \%-M E F V}, \dot{\mathrm{V}}_{\text {Emax60\%TLC-MEFV, }}, \dot{\mathrm{V}}_{\text {Emax-isovol.60\%TLC-MEFV, }}$ $\dot{\mathrm{V}}_{\mathrm{Emax} 25 \% \mathrm{VC}-\mathrm{MEFV}}$, and $\dot{\mathrm{V}}_{\text {Emax25\%VC-PEFV }}$ ). The limits of "normal" reactions, defined by decreases in mean percentage of baseline plus $2 \mathrm{SD}$ in the $\mathrm{HC}$ group were the following: $-9 \%$ for $\mathrm{FEV}_{1}$, $-21 \%$ for $\mathrm{FEF}_{25-75},-26 \%$ for $\mathrm{V}_{\text {Emax } 50 \% \mathrm{VC}-\mathrm{MEFV}},-31 \%$ for $\dot{\mathrm{V}}_{\text {Emax } 60 \% \text { TLC-MEFV }},-37 \%$ for $\dot{\mathrm{V}}_{\text {Emax-isovol.60\%TLC-MEFV }},-25 \%$ for $\dot{\mathrm{V}}_{\text {Emax } 25 \% \mathrm{VC}-\mathrm{MEFV}},-26 \%$ for $\dot{\mathrm{V}}_{\text {Emax } 25 \% \mathrm{VC}-\mathrm{PEFV}}$.

The responses measured $5 \mathrm{~min}$ after the challenges expressed in percentage of predicted values for the seven most discriminative parameters are shown in Table 3 . After all three challenges the highest degree of abnormality occurred in small volumerelated flow rates. When expressing the results in this way, there was some overlap between the two groups.

In Figure 1, correlations of baseline values with cold air induced changes, in percentage of baseline, for seven expiratory flows are shown for Group A. Most of these were not statistically significant. For $\mathrm{FEV}_{1}$ a significant negative correlation was found, suggesting a stronger reaction in those with more abnormal baseline values. The slopes of negative regression lines tended to be closer to horizontal for flow rates measured at lower lung volumes during the forced expiration, and for $\dot{V}_{\text {Emax } 25 \% \text { C.PEFV }} a$ significant positive correlation was found, indicating a weaker response in subjects with more abnormal baseline values.

The reproducibility of induced bronchoconstriction in repeated CAChs was evaluated by comparing changes in percentage of baseline and after challenge values in percentage of predicted. Results of CACh I and II were correlated for Groups A and HC combined, as well as for those 13 asthmatics alone who also participated in CACh III. In addition, long-term reproducibility was evaluated by comparing the results of CACh I and III. Figure 2 compares the reproducibility of changes and of after challenge values for $\mathrm{FEV}_{1}$ and $\dot{\mathrm{V}}_{\text {Emax25\%vC-MEFv. Table } 4 \text { demonstrates }}$ markedly higher correlation coefficients for the values in percentage of predicted than for the changes in percentage of baseline after $\mathrm{CACh}$, even exceeding the reproducibility of the baseline values. This table also shows a higher reproducibility of values obtained with $2 \mathrm{~h}$ than with 2 wk between CAChs.

Measurements $15 \mathrm{~min}$ after the $\mathrm{CACh}$ in Group A indicated different degrees of recovery from the reaction for different flow rates. Fifteen minutes after CACh I the change in $\dot{\mathrm{V}}_{\text {Emax25\%VC- }}$ MEFv was still $-33.3 \pm 23.9 \%$ of baseline whereas the change in $\mathrm{FEV}_{1}$ was $-16.2 \pm 14.0 \%$ of baseline. Thus children with asthma recovered about $50 \%$ of the original decrease in $\mathrm{FEV}_{1}$, but only about one-third in the other flows. Large interindividual differences in recovery were observed.

The temperature of inspired air was $-17.0 \pm 5.9^{\circ} \mathrm{C}$ (range, -5 to $-30^{\circ} \mathrm{C}$ ) in the first challenge for Group $\mathrm{A}$ and $-15.8 \pm$ $3.8^{\circ} \mathrm{C}$ (range, -9 to $-21^{\circ} \mathrm{C}$ ) for Group $\mathrm{HC}$. In six subjects of Group $A$ and in three of Group $\mathrm{HC}$, the air temperature was outside the range of -10 to $-20^{\circ} \mathrm{C}$. In the second challenge the temperatures were $-15.0 \pm 4.5^{\circ} \mathrm{C}\left(-9\right.$ to $\left.-26^{\circ} \mathrm{C}\right)$ for Group $\mathrm{A}$ and $-13.3 \pm 3.3^{\circ} \mathrm{C}\left(-8\right.$ to $\left.-21^{\circ} \mathrm{C}\right)$ for Group $\mathrm{HC}$, with two challenges in $\mathrm{A}$ and 2 in $\mathrm{HC}$ outside the target range. In the third challenge the temperatures were $-12.3 \pm 3.1^{\circ} \mathrm{C}\left(-7\right.$ to $\left.-18^{\circ} \mathrm{C}\right)$ with two outside the target range. In 16 children with asthma, two cold air provocations were performed on the same day. Except for one child who ventilated with identical inspiratory air temperatures in both procedures, all cold air-induced changes after $\mathrm{CACh}$ with the lower inspiratory air temperature were significantly different from those with the higher temperature for $\mathrm{FEV}_{1}(-31.2 \%$ baseline $\pm 14.8 \%$ versus $-26.3 \pm 14.9 \%, P<$ $0.01)$ and for $\dot{V}_{\text {Emax } 25 \% \mathrm{VC}-\mathrm{MEFV}}(-47.2 \%$ baseline $\pm 14.2 \%$ versus $-38.8 \pm 10.9 \%, P<0.05)$. $\mathrm{FEV}_{1}$ was also significantly smaller in terms of percentage predicted after $\mathrm{CACh}$ with the lower than 
Table 3. After challenge values* (5 min after $C A C h$ )

\begin{tabular}{|c|c|c|c|c|c|}
\hline & \multicolumn{2}{|c|}{ CACh I } & \multicolumn{2}{|c|}{$\mathrm{CACh}$ II } & \multirow{2}{*}{ 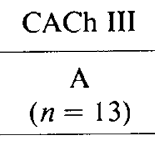 } \\
\hline & $\begin{array}{c}\mathrm{A} \\
(n=22)^{*}\end{array}$ & $\begin{array}{c}\mathrm{HC} \\
(n=18)\end{array}$ & $\begin{array}{c}\mathrm{A} \\
(n=16)\end{array}$ & $\begin{array}{c}\mathrm{HC} \\
(n=15)\end{array}$ & \\
\hline $\begin{array}{l}\mathrm{FEV}_{1} \\
\quad(\% \text { predicted })\end{array}$ & $\begin{array}{l}60.0 \pm 20.1 \\
(14-97)\end{array}$ & $\begin{array}{r}95.3 \pm 9.3 \\
(79-112)\end{array}$ & $\begin{array}{c}64.7 \pm 20.9 \\
(24-105)\end{array}$ & $\begin{array}{r}93.8 \pm 9.5 \\
(78-111)\end{array}$ & $\begin{array}{c}54.8 \pm 23.7 \\
(17-109)\end{array}$ \\
\hline $\begin{array}{l}\mathrm{FEF}_{25-75} \\
\quad(\% \text { predicted })\end{array}$ & $\begin{array}{c}33.9 \pm 20.1 \\
(9-81)\end{array}$ & $\begin{array}{c}92.3 \pm 15.6 \\
(59-112)\end{array}$ & $\begin{array}{l}37.2 \pm 20.1 \\
(10-76)\end{array}$ & $\begin{array}{c}86.7 \pm 15.6 \\
(56-108)\end{array}$ & $\begin{array}{c}31.8 \pm 23.4 \\
(9-89)\end{array}$ \\
\hline $\begin{array}{l}\dot{\mathrm{V}}_{\text {Emax } 50 \% \mathrm{VC}-\mathrm{MEFV}} \\
(\% \text { predicted })\end{array}$ & $\begin{array}{c}33.9 \pm 21.6 \\
(10-89)\end{array}$ & $\begin{array}{l}79.7 \pm 13.3 \\
(50-97)\end{array}$ & $\begin{array}{c}37.3 \pm 16.5 \\
(19-75)\end{array}$ & $\begin{array}{l}74.3 \pm 14.8 \\
(49-96)\end{array}$ & $\begin{array}{c}30.8 \pm 22.0 \\
(9-85)\end{array}$ \\
\hline $\begin{array}{r}\dot{\mathrm{V}}_{\text {Emax60\%TLC-MEFV }} \\
(\% \text { predicted })\end{array}$ & $\begin{array}{c}30.4 \pm 22.5 \\
(3-87)\end{array}$ & $\begin{array}{c}84.7 \pm 15.5 \\
(44-103)\end{array}$ & $\begin{array}{c}34.9 \pm 19.1 \\
(11-77)\end{array}$ & $\begin{array}{c}79.9 \pm 16.1 \\
(53-108)\end{array}$ & $\begin{array}{c}28.2 \pm 22.8 \\
(6-83)\end{array}$ \\
\hline $\begin{array}{l}\dot{\mathrm{V}}_{\text {Emax-isovol. } 60 \% \mathrm{TLC}-\mathrm{MEFV}} \\
\text { (\% predicted) }\end{array}$ & $\begin{array}{c}30.1 \pm 21.3 \\
(5-81)\end{array}$ & $\begin{array}{c}87.9 \pm 15.6 \\
(49-119)\end{array}$ & $\begin{array}{l}31.8 \pm 18.6 \\
(12-77)\end{array}$ & $\begin{array}{c}78.7 \pm 16.8 \\
(53-108)\end{array}$ & $\begin{array}{c}23.5 \pm 16.4 \\
(4-58)\end{array}$ \\
\hline $\begin{array}{l}\dot{\mathrm{V}}_{\text {Emax25\%VC-MEFV }} \\
(\% \text { predicted })\end{array}$ & $\begin{array}{c}27.1 \pm 17.1 \\
(7-67)\end{array}$ & $\begin{array}{c}77.3 \pm 13.7 \\
(50-99)\end{array}$ & $\begin{array}{l}28.5 \pm 15.0 \\
(12-63)\end{array}$ & $\begin{array}{l}70.9 \pm 15.5 \\
(44-91)\end{array}$ & $\begin{array}{c}24.5 \pm 19.0 \\
(9-71)\end{array}$ \\
\hline $\begin{array}{l}\dot{\mathrm{V}}_{\text {Emax } 25 \% \mathrm{VC}-\mathrm{PEFV}} \\
\text { (\% predicted) }\end{array}$ & $\begin{array}{c}24.4 \pm 10.2 \\
(10-44)\end{array}$ & $\begin{array}{c}67.0 \pm 15.9 \\
(43-102) \\
\end{array}$ & $\begin{array}{c}24.9 \pm 14.3 \\
(8-52)\end{array}$ & $\begin{array}{c}62.6 \pm 13.3 \\
(42-80)\end{array}$ & $\begin{array}{c}23.0 \pm 16.6 \\
(9-59) \\
\end{array}$ \\
\hline
\end{tabular}

$*$ Values are mean $\pm \mathrm{SD}$ (range).

$\dagger n=23$ for $\mathrm{FEV}_{1}$ and $\mathrm{FEF}_{25-75}$.

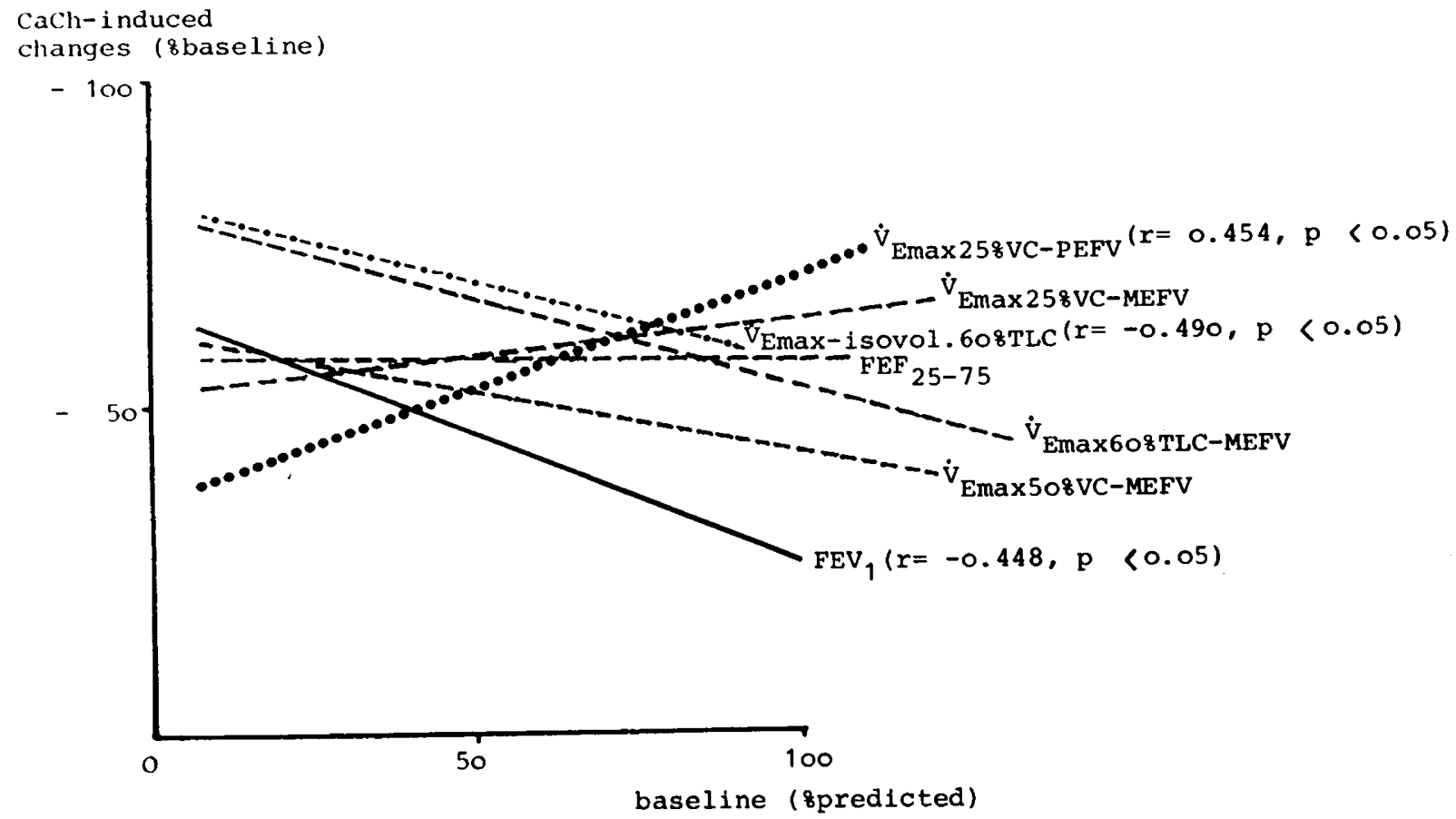

Fig. 1. Baseline response interrelations in CACh I. Changing behavior of regression lines $(n=22 \mathrm{~A})$. Correlation coefficients are only given for statistically significant correlations.

the higher inspiratory air temperature $(60.7 \pm 21.9 \%$ versus 65.4 $\pm 21.5 \%, P<0.05$ ), but this was not the case for $\dot{\mathrm{V}}_{\text {Emax } 25 \% \mathrm{VC}-\mathrm{MEFV}}$ $(28.9 \pm 18.7 \%$ versus $29.5 \pm 15.1 \%)$.

After the first $\mathrm{CACh}$, six children of Group A denied any sensation of tightness in the chest. $\mathrm{FEV}_{1}$ changes in this subgroup ranged from -12 to $-22 \%$ of baseline. In 10 asthmatics grading their airway obstruction as "mild," the change of FEV , was -17 to $-53 \%$, in six "moderate" -17 to $-52 \%$ and in one child who experienced dyspnea it was $-65 \%$ of baseline. The latter was the only child with a baseline $\mathrm{FEV}_{1}$ under $60 \%$ of predicted. Fifteen minutes after the first CACh only one child felt a moderate, and six a mild tightness in the chest.

The response to the bronchodilator aerosol was assessed in 20 children of Group A. Eight were mediated at the end of the first day of testing, seven after the second, and five after both. Fifteen minutes after the inhalation, $\mathrm{FEV}_{1}$ was $92.1 \pm 19.2 \%$ of predicted (range, $47-126 \%$ ), $\mathrm{FEF}_{25-75}$ was $87.6 \pm 31.9 \%$ of predicted (21$150 \%), \dot{V}_{\text {Emax } 50 \% V C-M E F V}$ was $79.4 \pm 25.6 \%$ of predicted $(22-$ $127 \%), \dot{V}_{\text {Emax } 25 \% \mathrm{VC}-\mathrm{MEFV}}$ was $67.9 \pm 32.2 \%$ of predicted (16$154 \%$ ), and $\dot{V}_{E \max 25 \% \mathrm{VC}-\mathrm{PEFV}}$ was $70 \pm 31.6 \%$ of predicted (18$155 \%)$. Evidently expiratory airflows did not return to normal in every child. When all childrens' baseline values were expressed as percentages of postbronchodilator values and compared with the cold air-induced changes, in percentage of baseline, no significant correlation was found, except for $\mathrm{FEV}_{1}$ in the first $\mathrm{CACh}$ $(r=0.498, \mathrm{SE}=13.60, P<0.05)$.

Means of PEF-recordings during the week after the first $\mathrm{CACh}$ ranged from $76-135 \%$ of predicted with coefficients of variation of 2 to $35 \%$. After the third CACh and the $\mathrm{HCh}$ mean PEFs were 78 to $132 \%$ of predicted and the coefficients of variation 3 to $31 \%$. Both mean PEFs and the coefficients of variation correlated better with the after challenge values of $\mathrm{FEV}_{1}$ and 

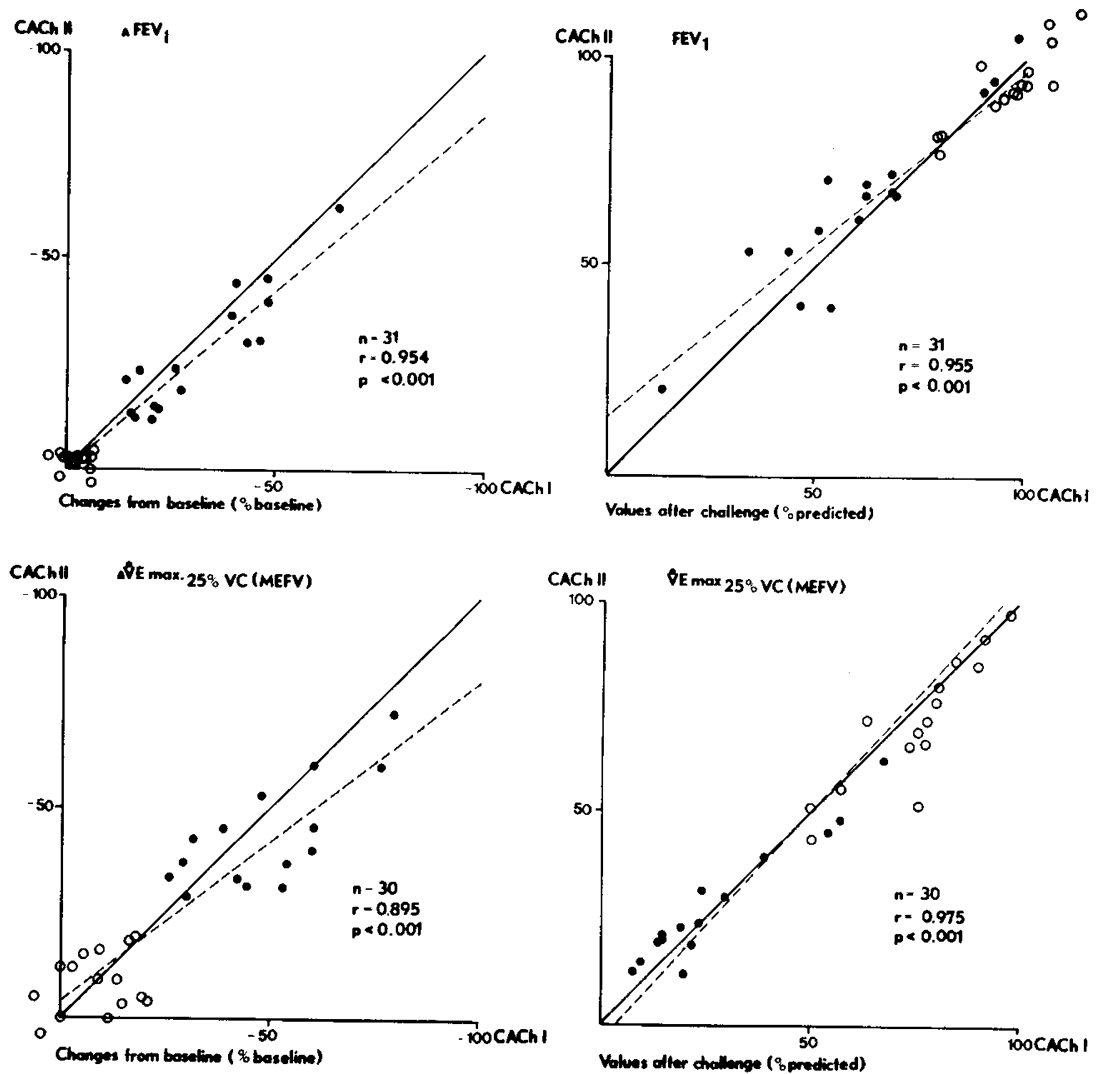

Fig. 2. Reproducibility of induced changes (\% baseline) and of after challenge values (\% predicted). No difference in the reproducibility for $\mathrm{FEV}_{1}$. Markedly higher reproducibility of $\%$ of predicted values after challenge than of changes in $\%$ of baseline for $\dot{\mathrm{V}}_{\text {Emax } 25 \% \mathrm{~V}-\mathrm{MEFv}}$ ( Closed circles) children with asthma; (Open circles) healthy control children; (----) regression line; and (- $($ ) line of identity.

Table 4. Reproducibility*

\begin{tabular}{|c|c|c|c|}
\hline & $\begin{array}{c}\text { Correlation } \\
\text { CACh I-CACh II } \\
{[n=30 \dagger(15 \mathrm{~A}+15 \mathrm{HC})]}\end{array}$ & $\begin{array}{c}\text { Correlation } \\
\text { CACh I-CACh II } \\
(n=13 A \ddagger)\end{array}$ & $\begin{array}{c}\text { Correlation } \\
\text { CACh I-CACh III } \\
(n=13 \mathrm{~A})\end{array}$ \\
\hline $\begin{array}{l}\triangle \mathrm{FEV}_{1} \\
\mathrm{FEV}_{1} \text { after } \mathrm{CACh} \\
\text { Baseline } \mathrm{FEV}_{1}\end{array}$ & $\begin{array}{l}r=0.954, P<0.001 \\
r=0.955, P<0.001 \\
r=0.839, P<0.001\end{array}$ & $\begin{array}{l}r=0.868, P<0.001 \\
r=0.900, P<0.001 \\
r=0.671, P<0.02\end{array}$ & $\begin{array}{l}r=0.571, P<0.05 \\
r=0.824, P<0.001 \\
r=0.808, P<0.001\end{array}$ \\
\hline $\begin{array}{l}\Delta \mathrm{FEF}_{25-75} \\
\mathrm{FEF}_{25-75} \text { after } \mathrm{CACh} \\
\text { Baseline } \mathrm{FEF}_{25-75}\end{array}$ & $\begin{array}{l}r=0.883, P<0.001 \\
r=0.948, P<0.001 \\
r=0.868, P<0.001\end{array}$ & $\begin{array}{l}r=0.702, P<0.01 \\
r=0.916, P<0.001 \\
r=0.740, P<0.01\end{array}$ & $\begin{array}{l}r=0.401, \mathrm{NS} \\
r=0.812, P<0.001 \\
r=0.772, P<0.01\end{array}$ \\
\hline $\begin{array}{l}\Delta \dot{\mathrm{V}}_{\text {Emax50\%VC-MEFV }} \\
\dot{\mathrm{V}}_{\text {Emax } 50 \% \mathrm{VC}-\mathrm{MEFV} \text { after CACh }} \\
\text { Baseline } \dot{\mathrm{V}}_{\text {Emax } 50 \% \mathrm{VC}-\mathrm{MEFV}}\end{array}$ & $\begin{array}{l}r=0.903, P<0.001 \\
r=0.963, P<0.001 \\
r=0.820, P<0.001\end{array}$ & $\begin{array}{l}r=0.713, P<0.01 \\
r=0.955, P<0.001 \\
r=0.776, P<0.01\end{array}$ & $\begin{array}{l}r=0.429, \mathrm{NS} \\
r=0.888, P<0.001 \\
r=0.724, P<0.01\end{array}$ \\
\hline $\begin{array}{l}\Delta \dot{V}_{\text {Emax60\%TLC-MEFV }} \\
\dot{\mathrm{V}}_{\text {Emax60\%TLC-MEFV after CACh }} \\
\text { Baseline } \dot{\mathrm{V}}_{\text {Emax60\% TLC-MEFV }}\end{array}$ & $\begin{array}{l}r=0.870, P<0.001 \\
r=0.926, P<0.001 \\
r=0.858, P<0.001\end{array}$ & $\begin{array}{l}r=0.709, P<0.01 \\
r=0.950, P<0.001 \\
r=0.784, P<0.01\end{array}$ & $\begin{array}{l}r=0.079, \mathrm{NS} \\
r=0.880, P<0.001 \\
r=0.683, P<0.02\end{array}$ \\
\hline $\begin{array}{l}\Delta \dot{\mathrm{V}}_{\text {Emax-isovol.60\%TLC-MEFV }} \\
\dot{\mathrm{V}}_{\text {Emax-isovol.60\%TLC after CACh }}\end{array}$ & $\begin{array}{l}r=0.867, P<0.001 \\
r=0.937, P<0.001\end{array}$ & $\begin{array}{l}r=0.727, P<0.01 \\
r=0.957, P<0.001\end{array}$ & $\begin{array}{l}r=0.160, \mathrm{NS} \\
r=0.845, P<0.001\end{array}$ \\
\hline $\begin{array}{l}\Delta \dot{\mathrm{V}}_{\text {Emax25\%VC-MEFV }} \\
\dot{\mathrm{V}}_{\text {Emax25\%VC-MEFV after CACh }} \\
\text { Baseline } \dot{\mathrm{V}}_{\text {Emax25\%VC-MEFV }}\end{array}$ & $\begin{array}{l}r=0.895, P<0.001 \\
r=0.975, P<0.001 \\
r=0.884, P<0.001\end{array}$ & $\begin{array}{l}r=0.797, P<0.01 \\
r=0.961, P<0.001 \\
r=0.869, P<0.001\end{array}$ & $\begin{array}{l}r=0.449, \mathrm{NS} \\
r=0.855, P<0.001 \\
r=0.754, P<0.1\end{array}$ \\
\hline $\begin{array}{l}\Delta \dot{V}_{\text {Emax } 25 \% \mathrm{VC}-\mathrm{PEFV}} \\
\dot{\mathrm{V}}_{\text {Emax } 25 \% \mathrm{VC}-\mathrm{PEFV} \text { after CACh }} \\
\text { Baseline } \dot{\mathrm{V}}_{\text {Emax } 25 \% \mathrm{VC}-\mathrm{PEFV}}\end{array}$ & $\begin{array}{l}r=0.815, P<0.001 \\
r=0.960, P<0.001 \\
r=0.785, P<0.001\end{array}$ & $\begin{array}{l}r=0.606, P<0.05 \\
r=0.889 P<0.001 \\
r=0.700, P<0.01\end{array}$ & $\begin{array}{l}r=0.040, \mathrm{NS} \\
r=0.628, P<0.05 \\
r=0.630, P<0.05\end{array}$ \\
\hline
\end{tabular}

* Abbreviations: NS, not significant and see "Abbreviations."

$\dagger n=31(16 \mathrm{~A}+15 \mathrm{HC})$ for $\mathrm{FEV}_{1}, \mathrm{FEF}_{25-75}$, and all baseline values.

$\ddagger$ Evaluation restricted to those $13 \mathrm{~A}$ who participated in CaCh III. 
$\dot{\mathrm{V}}_{\mathrm{Emax} 25 \% \mathrm{VC}-\mathrm{MEFV}}$ in percentage of predicted than with the challenge-induced changes in percentage of baseline (Table 5).

Eighteen children with asthma underwent an $\mathrm{HCh}$. In each case a CACh was done on the same day. Eleven times the $\mathrm{CACh}$ was done first, seven times the $\mathrm{HCh}$. All children responded positively to histamine as defined by at least a $20 \%$ baseline fall in $\mathrm{FEV}_{1}$. For the whole group $\mathrm{PC}_{20 \text {-histamine was }} 2.06 \pm 2.79 \mathrm{mg} /$ $\mathrm{ml}$ (range, $0.28-10.0 \mathrm{mg} / \mathrm{ml}$ ) (Table 6). The $\mathrm{PC}_{20 \text {-histamine }}$ values did.not correlate with either cold air-induced changes, or with post-CACh values in percentage of predicted, excepting those for $\mathrm{FEV}_{1}(r=0.496, \mathrm{SE}=20.06, P<0.05)$.

Recovery after $\mathrm{HCh}$ was highly variable. Fifteen minutes after the completion of the procedure, $\mathrm{FEV}_{1}$ was still $5.7 \pm 10.8 \%$ (range, +15 to $-31 \%$ ) below the baseline. The time for recovery to $95 \%$ of prechallenge $\mathrm{FEV}_{1}$ was $17.4 \pm 9.0 \mathrm{~min}$ ( 7 to $42 \mathrm{~min}$ ).

In contrast to the results after $\mathrm{CACh}, \mathrm{PC}_{20 \text {-histamine }}$ did not correlate with either the mean or with the coefficient of variation of the PEF values recorded during the week after testing. In those five children of Group A who had CAChs done both with and without a preceding medication break (three being treated with chromolyn sodium and two with a combination of an inhaled sympathomimetic, a topical steroid and an oral theophylline) the cold air-induced changes and the post-CACh abnormalities were invariably larger when unmedicated.

\section{DISCUSSION}

The results of this study prove that $\mathrm{CACh}$ carries a high potential for practical use in children. When the cold air-induced alterations were expressed in percentage of baseline, several expiratory flow measurements $\left(\mathrm{FEV}_{1}, \mathrm{FEF}_{25-75}, \dot{\mathrm{V}}_{\mathrm{Emax} 50 \% \mathrm{VC}-\mathrm{MEFV}}\right.$,
$\dot{\mathrm{V}}_{\text {Emax60\%TLC-MEFV }}, \quad \dot{\mathrm{V}}_{\text {Emax-isovol.60\%TLC-MEFV }}, \quad \dot{\mathrm{V}}_{\text {Emax 25\%VC-MEFV }}$, $\dot{\mathrm{V}}_{\text {Emax } 25 \% \mathrm{VC}-\mathrm{PEFV}}$ ) clearly distinguished between healthy children and those with asthma. The limits of repsonses in healthy subjects found in this study are outside the average intrasubject variability of the appropriate PFT measurements (21), and they closely approximate the corresponding limits for exercise bronchoprovocation testing in children (23). Such power of discrimination was also found for adults (17). The no overlap of cold air-induced responses between groups in this study is most likely attributable to strict selection criteria for both groups. In an unselected pediatric population an intermediate group between healthy and clearly hyperreactive individuals, such as in the case of adult hay fever patients (17) could exist.

TLC only increased to a minor extent after CACh. Consequently $\dot{\mathrm{V}}_{\text {Emax-isovol.60\%TLC-MEFV }}$ did not differ from $\dot{\mathrm{V}}_{\text {Emax60\%TLC }}$ MEFV. As FVC showed a general tendency to decrease after $\mathrm{CACh}$, $\dot{\mathrm{V}}_{\mathrm{Emax}}$ measured at various isovolume points of prechallenge FVC would have rendered the induced changes as well as postCACh abnormalities more rather than less conspicuous.

The fact that partial and maximal flow-volume curves were comparable suggests that the volume history of the lung, which theoretically could influence the results $(19,30)$, has little relevance in the clinical use of the method. The minor decrease of inspiratory airflow in the asthmatics supports the previous observation that children with recurrent croup do but those with asthma do not demonstrate significant inspiratory flow limitation after histamine inhalation (43).

As heat flux from the airway surface depends on convective as well as evaporative losses, respiratory heat exchange is enhanced both by low temperature and water content of the inhaled air $(13,14,39)$. In the present study air was not dried before cooling

Table 5. Correlation CACh-peak flow recording*

\begin{tabular}{|c|c|c|c|c|}
\hline & \multicolumn{2}{|c|}{$\begin{array}{c}\text { Correlation CACh I-PEF after visit } 1 \\
\left(n=23 \mathrm{~A} \text { for } \mathrm{FEV}, 22 \mathrm{~A} \text { for } \dot{\mathrm{V}}_{\mathrm{Emax} 25 \% \mathrm{VC}-\mathrm{MEFV}}\right)\end{array}$} & \multicolumn{2}{|c|}{$\begin{array}{l}\text { Correlation CACh III-PEF after visit } 2 \\
\qquad(n=13 \mathrm{~A})\end{array}$} \\
\hline & $\begin{array}{l}\text { Mean PEF } \dagger \\
\text { (\% predicted) }\end{array}$ & $\begin{array}{l}\mathrm{CV} \ddagger \\
(\%)\end{array}$ & $\begin{array}{l}\text { Mean PEF } \\
(\% \text { predicted })\end{array}$ & $\begin{array}{l}\mathrm{CV} \\
(\%)\end{array}$ \\
\hline $\begin{array}{l}\Delta \mathrm{FEV} \\
(\% \text { baseline })\end{array}$ & $\begin{array}{l}r=-0.356 \\
\text { NS }\end{array}$ & $\begin{array}{l}r=0.519 \\
P<0.02\end{array}$ & $\begin{array}{l}r=-0.174 \\
\mathrm{NS}\end{array}$ & $\begin{array}{l}r=0.483 \\
\text { NS }\end{array}$ \\
\hline $\begin{array}{l}\mathrm{FEV}_{1} \text { after } \mathrm{CACh} \\
\text { (\% predicted) }\end{array}$ & $r=0.462$ & $r=-0.529$ & $r=0.556$ & $r=-0.687$ \\
\hline $\begin{array}{c}\text { (\% predicted) } \\
\Delta \dot{\mathrm{V}}_{\mathrm{E}} 2 x 5 \% \mathrm{VCMEFV}\end{array}$ & $P<0.05$ & $P<0.02$ & $P<0.05$ & $P<0.01$ \\
\hline $\begin{array}{l}\Delta \dot{\mathrm{V}}_{\text {Emax } 25 \% \mathrm{VC}-\mathrm{MEFV}} \\
\quad \text { (\% baseline })\end{array}$ & $\begin{array}{l}r=-0.120 \\
\mathrm{NS}\end{array}$ & $\begin{array}{l}r=0.361 \\
\text { NS }\end{array}$ & $\begin{array}{l}r=0.047 \\
\text { NS }\end{array}$ & $\begin{array}{l}r=0.153 \\
\text { NS }\end{array}$ \\
\hline $\begin{array}{l}\dot{V}_{\text {Emax25\%VC-MEFV after CACh }} \\
\text { (\% predicted) }\end{array}$ & $\begin{array}{l}r=0.435 \\
P<0.05\end{array}$ & $\begin{array}{l}r=-0.550 \\
P<0.01\end{array}$ & $\begin{array}{l}r=0.452 \\
\mathrm{NS}\end{array}$ & $\begin{array}{l}r=-0.575 \\
P<0.05\end{array}$ \\
\hline
\end{tabular}

*Abbreviations, see "Abbreviations."

$\dagger$ Mean of 14 measurements done in 1 wk.

$\ddagger$ Coefficient of variation of 14 measurements.

Table 6. Histamine inhalation challenge*

\begin{tabular}{|c|c|c|c|}
\hline & $\begin{array}{c}\text { Baseline values } \\
(\% \text { predicted })\end{array}$ & $\begin{array}{l}\text { Histamine-induced changes } \\
(\% \text { baseline })\end{array}$ & $\begin{array}{c}\text { Values } 3 \text { min after } \mathrm{HCh} \\
(\% \text { predicted })\end{array}$ \\
\hline$\overline{F E V}_{1}$ & $\begin{array}{c}80.6 \pm 16.9 \\
(60-126)\end{array}$ & $\begin{array}{l}-33.4 \pm 8.8 \\
(-20 \text { to }-54)\end{array}$ & $\begin{array}{l}52.9 \pm 15.9 \\
(20-96)\end{array}$ \\
\hline $\mathrm{FEF}_{25-75}$ & $\begin{array}{c}60.3 \pm 28.9 \\
(18-123)\end{array}$ & $\begin{array}{l}-57.6 \pm 12.8 \\
(-22 \text { to }-74)\end{array}$ & $\begin{array}{l}24.8 \pm 11.9 \\
\quad(7-58)\end{array}$ \\
\hline$\dot{\mathrm{V}}_{\mathrm{Emax} 50 \% \mathrm{VC}-\mathrm{MEFV}}$ & $\begin{array}{c}56.4 \pm 24.9 \\
(22-107)\end{array}$ & $\begin{array}{l}-38.4 \pm 13.6 \\
(-13 \text { to }-62)\end{array}$ & $\begin{array}{c}33.9 \pm 16.1 \\
\quad(15-77)\end{array}$ \\
\hline$\dot{\mathrm{V}}_{\text {Emax60\%TLC-MEFV }}$ & $\begin{array}{c}56.3 \pm 29.3 \\
(11-108)\end{array}$ & $\begin{array}{l}-46.4 \pm 14.1 \\
(-19 \text { to }-70)\end{array}$ & $\begin{array}{c}30.6 \pm 16.6 \\
\quad(4-62)\end{array}$ \\
\hline$\dot{\mathrm{V}}_{\text {Emax-isovol.60\%TLC-MEFV }}$ & $\ldots$ & $\begin{array}{l}-46.9 \pm 13.0 \\
(-22 \text { to }-65)\end{array}$ & $\begin{array}{l}29.3 \pm 15.5 \\
\quad(7-62)\end{array}$ \\
\hline$\dot{\mathrm{V}}_{\mathrm{Emax} 25 \% \mathrm{VC}-\mathrm{MEFV}}$ & $\begin{array}{l}48.9 \pm 24.6 \\
(20-99)\end{array}$ & $\begin{array}{l}-45.8 \pm 13.8 \\
(-25 \text { to }-75)\end{array}$ & $\begin{array}{c}24.5 \pm 11.0 \\
(11-55)\end{array}$ \\
\hline$\dot{\mathrm{V}}_{\mathrm{Emax} 25 \% \mathrm{VC}-\mathrm{PEFV}}$ & $\begin{array}{c}44.3 \pm 21.1 \\
(18-85)\end{array}$ & $\begin{array}{r}-40.1 \pm 19.0 \\
(-7 \text { to }-75)\end{array}$ & $\begin{array}{c}23.8 \pm 11.3 \\
(6-44)\end{array}$ \\
\hline
\end{tabular}

\footnotetext{
*Values are mean $\pm \mathrm{SD}$ (range).
} 
for convenience's sake, but the absolute water content of air at sub-zero temperatures is so low that evaporation-dependent heat loss must have been maximal.

Abnormal responses to cold-air provocation were not restricted to children with a history of exercise-induced asthma, although the test can, in part, be viewed as a model for it (18). Previous laboratory studies on the incidence of this phenomenon in children produced inconsistent results, probably due to uncontrolled climatic factors $(3,23,24,29,36)$. This has limited the practical usefulness of even standardized exercise bronchoprovocation $(12,36)$ for the assessment of airway hyperreactivity in children (29). In the present study all 23, randomly selected, patients of a pediatric asthma clinic demonstrated a reaction to cold air hyperventilation which exceeded that of healthy control subjects. Airway hyperreactivity to induced respiratory heat loss apparently is, thus, a general phenomenon in childhood asthma, with a sensivity superior to exercise. Some investigators suggested a "temperature-dependent" group of children reacting mainly to heat loss, and an "exercise-dependent" one reacting predominantly to exercise per se $(4,5)$. This was explained by assuming that in the "temperature-dependent" group airway cooling affects mast cells located in more central airways, whereas exercise affects the more peripherally located ones (5). In the present study, much colder inspiratory air temperatures were reached than in the experiments leading to the above theory, thus, respiratory heat loss will have penetrated deeper into the tracheobronchial tree, thereby reaching peripheral as well as central target systems.

Only about one-third of the patients reacted excessively to outdoor exercise in the winter. Theoretically this apparent contradiction could be explained by an adaptation to cold air after prolonged exposure. Children could also have a limited and variable perception of their airflow limitation, such as found in adult asthmatics $(7,27)$. This possibility is supported by considerable interindividual differences in the subjective grading to chest tightness in the present study.

Evidence of a predominant contribution of small airways to cold air-induced expiratory airflow obstruction is given by greater changes in small airway-related flow rates. This predominant role of small airway obstruction can also be seen in the mean baseline values, which decreased towards the end of forced expiration, and in the effect of a bronchodilator medication which was least complete as measured by $\dot{\mathrm{V}}_{\mathrm{Emax} 25 \% \mathrm{vC}}$. Furthermore, flow rates late during forced expiration recovered slower after the provocation than FEV . Others found long-lasting obstruction of the peripheral airways acting as a precondition for further asthmatic attacks (27). In asthmatic children, characteristic impairment of flows measured late during forced expiration was also shown $(20,44)$; thus, cold air-induced airway reaction seems to accurately reflect localized events of the clinical disorder, rendering it an appropriate model for studying the disease.

The occurrence of considerable small airway reaction without major discomfort might, in part, be explained by a relatively small concomitant reaction in the large airways. Others have also observed that subjective complaints are predominantly dependent on large airway obstruction (27).

The present study demonstrated different baseline-to-response correlations for different flow measurements. A negative correlation was found for $\mathrm{FEV}_{1}$ but a positive one for $\dot{\mathrm{V}}_{\mathrm{Emax} 25 \% \mathrm{VC}-\mathrm{PEFV} \text {. }}$ This tendency for FEV 1 might, in part, be the arithmetic result of relating a given absolute decrease of $F E V_{1}$ to a varying baseline value. But, the opposite tendency for $\dot{V}_{\text {Emax } 25 \% \mathrm{VC}-\mathrm{PEFV} \text {, which }}$ changed relatively less in patients with a lower baseline, indicates the existence of some "reaction-limiting" mechanism. Others have also speculated on such mechanisms when studying histamine challenge (10).

The results of cold air provocation in this study were expressed both as changes in percentage of baseline, and as postchallenge abnormalities in percentage of predicted values. Maximum flows recorded towards the end of the forced expiration were markedly more reproducible in form of the latter than of the former expression. This reproducibility even exceeded the one of some baseline measurements. The same was true when comparing CACh I and III. This again indicated an individual limitation of the reaction, most likely determined by some characteristics of the peripheral airways. The poorer reproducibility of results over a 2-wk period indicates spontaneous changes of reactivity.

In asthmatic adults a dose-response relationship for respiratory heat exchange and expiratory airflow changes was found (31). In the children studied here, respiratory heat exchange was not accurately quantified, as the expired air temperature was not measured; however, for $\mathrm{FEV}_{1}$, a trend towards lower values after $\mathrm{CACh}$ with the colder inspired air suggested such a dose-response relationship. No such trend was found for maximum flow at low lung volume $\left(\dot{\mathrm{V}}_{\text {Emax } 25 \% \mathrm{VC}-\mathrm{MEFV}}\right)$. Baseline-to-response correlations, reproducibility characteristics, and temperature-to-response interrelations all indicate a different dose-response behavior for different flow measurements. The cold air-induced change of $\mathrm{FEV}_{1}$ apparently took place on the steep mid-portion of its doseresponse curve, resulting in more obstruction after a large dose. In contrast, the small airway-related flows were most likely shifted to the flatter upper part of a sigmoid dose-response curve. There, variations of respiratory heat exchange would not have a major effect. This divergent behavior of large and small airwayrelated flows could be explained by either a steeper mid-portion of the small-airway dose-response curve, or by a more obstructed baseline situation in the periphery, or both. If the limit of doseresponse presumably reached by $\mathrm{CACh}$, represents a genuine maximum of small airway reaction, the determinants of this maximum remain unknown. Merely mechanical limitations should have resulted in better reproducibility of stronger reactions to CACh. If, however, the maximum obstruction is determined by individual and variable intrinsic responsiveness, a smaller CACh-induced obstruction in one child should be as reproducible as a larger one in another. Figure 2 , indicating that the scatter of points for less obstruction was not more than that for higher airflow limitation, seems to support the latter hypothesis. It may be assumed that the endogenously predetermined maximum of small airway obstruction is linked to the momentary limit of individual airway hyperreactivity. Indeed in this study the absolute short-range after-challenge abnormality is highly reproducible and independent of the variable baseline situation.

The total range of responsiveness in asthmatic children can not be estimated if their individual "normal" pulmonary functions are not known. Because predicted mean values by height cannot account for interindividual differences in post-CACh values expressed as percentage of predicted, overlaps of individual results between $\mathrm{A}$ and $\mathrm{HC}$ occurred. This can be explained by the following example: in an asthmatic (or hyperreactive) child with a baseline of $114 \%$ predicted for $\mathrm{FEV}_{1}$, a postchallenge response of $24 \%$ would produce a value of $87 \%$ of predicted. In a healthy child with a baseline of $91 \%$ of predicted, a $4 \%$ of baseline change would result in the same end-point. Clearly then an individual child's airway reactivity can only be determined by percentage of predicted after challenge values if the absolute baseline is known. The latter could be estimated by a functional optimum produced with a bronchodilator medication. Responses to challenge could then be expressed as percentages of this individual optimum. In the present study, this could not be done reliably because bronchodilator was administered after the challenges.

Airway hyperreactivity is frequently measured by challenges with histamine or metacholine. The procedures have been standardized $(8,11,35)$. The response is described by the drug concentration producing a predetermined change of lung function (e.g., a $20 \%$ fall of $\mathrm{FEV}_{1}$ ). The disadvantages of this methodology include the time consuming procedure requiring meticulously standardized aerosols (35), and the widely varying pattern of aerosol deposition $(33,41)$. As evident from the present study, 


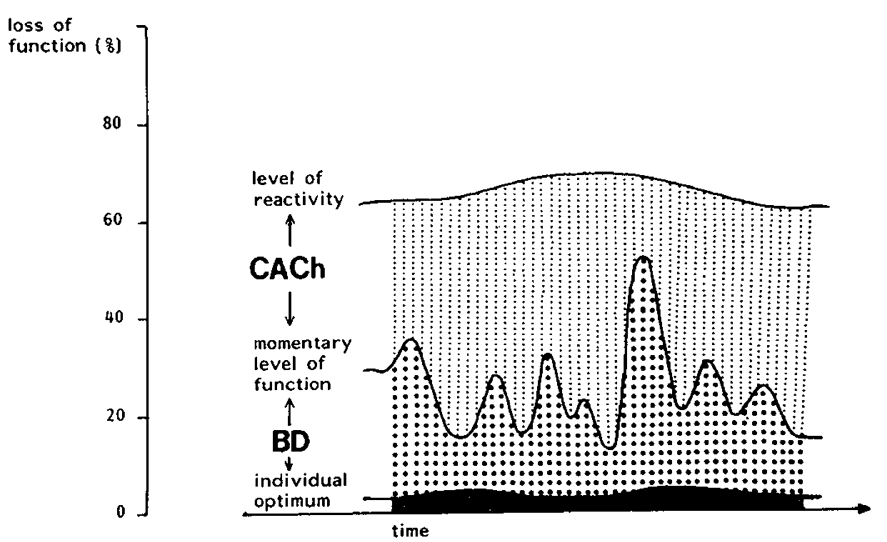

Fig. 3. The functional dimensions of childhood asthma (hypothetical). $\mathrm{BD}$, bronchodilator medication.

the time course of recovery after a positive reaction to histamine is highly variable. The response may not be related strictly to either the last inhaled drug concentration or to the accumulated dose of all inhalations (25). The complicated interrelation between baseline functions and response is poorly understood (10, 25 ). Drugs used in these inhalation challenges can, in high dosages, reduce lung function beyond the $20 \%$ limit in anyone, raising doubts about the specificity of such tests.

Although in a standardized inhalation challenge the highest bronchoconstrictor dose might cause a reaction greatly overshooting the target value, after $\mathrm{CACh}$ only one child showed marked and promptly reversible dyspnea. Children like this with a baseline FEV, below $60 \%$ of predicted should not be tested.

In comparison to pharmacologic challenges, $\mathrm{CACh}$ is less time consuming, void of problems with aerosol generation and deposition, yet it discriminates clearly between "normal" and hyperreactive individuals. It seems to be an acceptable model of childhood asthma and, for some lung functions, the response is independent from the baseline situation. Simulating hyperventilation on a cold winter day is also more physiologic and, thus, ethically more acceptable than the inhalation of pharmacologically active substances.

All children with asthma responded positively to both $\mathrm{CACh}$ and histamine but with no quantitative correlation of the results. In previous studies, close correlations between the responses to inhaled histamine and metacholine $(9,22)$, and between exercise and metacholine challenges $(9,26)$ were found. Opinions differ about the correlation of airway reactivity induced by histamine and exercise $(2,9,23)$.

The clinical usefulness of $\mathrm{CACh}$ is supported by the significant correlation found between the responses and PEF measurements during the week after the provocation. Such measurements did not correlate well with the results of the histamine challenge. Whether or not longer observation periods with PEF testing would have revealed a more accurate picture of the patient's condition, the observed differences between different phases of the study and between results expressed in different ways must be regarded as relevant. Cold air provocation might be superior to histamine challenge in characterizing the clinical severity of childhood asthma.

Five children who were tested when under medication seemed to be partially protected. Drug interaction with the response to $\mathrm{CACh}$ will have to be studied systematically together with the still controversial cellular, humoral, and neural mechanisms of the reaction $(5,6,18,32)$.

This new method of testing bronchial hyperreactivity, as adapted for use in pediatrics, can assist in the diagnosis of respiratory disturbances in children with atypical manifestations of asthma. In those with proven asthma a quantitative assessment of the level of reactivity could help in fine tuning of diagnostic and therapeutic decisions. It seems that cold air provocation can better characterize the physiologic abnormality than highly variable measurements of pulmomary functions (28). Using a bronchodilator for determining the child's individual functional optimum it is now possible to establish the whole range of "functional dimensions" of a child's asthma (Fig. 3). Whether, and to what extent the response of $\mathrm{CACh}$ is representative of responses to other triggering factors, including exercise, remains to be evaluated. At the present time one can only speculate about the nature and time course of the interrelations between baseline lung fucntions, a postbronchodilator tested optimum and the limits of reactivity.

\section{REFERENCES AND NOTES}

1. American Thoracic Society: Chronic bronchitis, asthma and pulmonary emphysema: definitions and classification of chronic bronchitis, asthma and pulmonary emphysema. Am. Rev. Respir. Dis., 85: 762 (1962).

2. Anderton, R. C., Cuff, M. T., Frith, P. A., Cockroft, D. W., Morse, J. L. C., Jones, N. L., and Hargreave, F. E.: Bronchial responsiveness to inhaled histamine and exercise. J. Allergy Clin. Immunol., 63: 315 (1979).

3. Balfour-Lynn, L., Tooley, M., and Godfrey, S.: Relationship of exerciseinduced asthma to clinical asthma in childhood. Arch. Dis. Child., 56: 450 (1981).

4. Ben-Dov, I., Bar-Yishay, E., and Godfrey, S.: Refractory period after exerciseinduced asthma unexplained by respiratory heat loss. Am. Rev. Respir. Dis., 125: 530 (1982).

5. Ben-Dov, I., Bar-Yishay, E., and Godfrey, S.: Heterogeneity in the response of asthmatic patients to pre-exercise treatment with cromolyn sodium. Am. Rev. Respir. Dis., 127: 113 (1983).

6. Breslin, F. J., McFadden, E. R., Jr., and Ingram, R. H., Jr.: The effects of cromolyn sodium on the airway response to hyperpnea and cold air in asthma. Am. Rev. Respir. Dis., 122: 11 (1980).

7. Burdon, J. G. W., Juniper, E. F., Killian, K. J., Hargreave, F. E., and Campbell, E. J. M.: The perception of breathlessness in asthma. Am. Rev. Respir. Dis., 126: 825 (1982)

8. Chai, H., Farr, R. S., Froehlich, L. A., Mathison, D. A., McLean, J. A., Rosenthal, R. R. Sheffer, A. L., Spector, S. L., and Townley, R. G.: Standardization of bronchial inhalation challenge procedures. J. Allergy Clin. Immunol., 56: 323 (1975).

9. Chatham, M., Blecker, E. R., Smith, P. L., Rosenthal, R. R., Mason, P., and Norman, P. S.: A comparison of histamine, metacholine, and exercise airway reactivity in normal and asthmatic subjects. Am. Rev. Respir. Dis., 126: 235 (1982).

10. Chung, K. F., Morgan, B., Keyes, S. J. and Snashall, P. D.: Histamine doseresponse relationships in normal and asthmatic subjects. The importance of starting airway caliber. Am. Rev. Respir. Dis., 126: 849 (1982).

11. Cockroft, D. W., Killian, D. N., Mellon, J. J. A., and Hargreave, F. E.: Bronchial reactivity to inhaled histamine: a method and clinical survey. Clin. Allergy, 7: 235 (1977).

12. Cropp, G. J. A.: The exercise bronchoprovocation test: standardization of procedures and evaluation of response. J. Allergy Clin. Immunol., 64: 627 (1979).

13. Deal, E. C., Jr., McFadden, E. R., Jr., Ingram, R. H., Jr., and Jaeger, J. J.: Effects of atropine on potentiation of exercise-induced bronchospasm by cold air. J. Appl. Physiol.: Respirat. Environ. Exercise Physiol., 45: 238 (1978).

14. Deal. E. C., Jr., McFadden, E. R., Jr., Ingram, R. H., Jr., Strauss, R. H., and Jaeger, J. J.: Role of respiratory heat exchange in production of exerciseinduced asthma. J. Appl. Physiol.: Respirat. Environ. Exercise Physiol., 46: 467 (1979)

15. Deal, E. C., Jr., McFadden, E. R., Jr., Ingram, R. H., Jr., and Jaeger, J. J.: Hyperpnea and heat flux: initial reaction sequence in exercise-induced asthma. J. Appl. Physiol.: Respirat. Environ. Exercise Physiol., 46: 476 (1979).

16. Deal, E. C., Jr., McFadden, E. R., Jr., Ingram, R. H., Jr., and Jaeger, J. J.: Esophageal temperature during exercise in asthmatic and nonasthmatic subjects. J. Appl. Physiol.: Respirat. Environ. Exercise Physiol., 46: 484 (1979).

17. Deal, E. C., Jr., McFadden, E. R., Jr., Ingram, R. H., Jr., Breslin, F. J., and Jaeger, J. J.: Airway responsiveness to cold air and hyperpnea in normal subjects and in those with hay fever and asthma. Am. Rev. Respir. Dis., 121: $621(1980)$

18. Deal, E. C., Jr., Wasserman, S. I., Soter, N. A., Ingram, R. H., and McFadden, E. R.: Evaluation of role played by mediators of immediate hypersensitivity in exercise-induced asthma. J. Clin. Invest., 65: 659 (1980).

19. Fish, J. E., Peterman, V. I., and Cugell, D. W.: Effect of deep inspiration on airway conductance in subjects with allergic rhinitis and allergic asthma. J. Allergy Clin. Immunol., 60: 41 (1977).

20. Hill, D. J., Landau, L. I., McNicol, K. N., and Phelan, P. D.: Asthma-the physiological and clinical spectrum in childhood. Respiratory function studies in its assessment. Arch. Dis. Child., 47: 874 (1972).

21. Hutchinson, A. A., Erben, A., McLennan, L. A., Landau, L. I., and Phelan, P. D.: Intrasubject variability of pulmonary function testing in healthy children. Thorax, 36: 370 (1981). 
22. Juniper, E. F., Frith, P. A., Dunnett, C., Cockroft, D. W., and Hargreave, F. E.: Reproducibility and comparison of responses to inhaled histamine and metacholine. Thorax, 33: 705 (1978)

23. Kattan, M., Keens, T. G., Mellis, C. M., and Levison, H.: The response to exercise in normal and asthmatic children. J. Pediatr., 92: 718 (1978).

24. Kawabori, I., Pierson, W. E., Conquest, L. L., and Bierman, C. W.: Incidence of exercise-induced asthma in children. J. Allergy Clin. Immunol., 58: 447 (1976).

25. Kerrebijn, K. F. and Neijens, H. J.: Measurement of the bronchial responsiveness in children. Progr. Respir. Res., 17: 143 (1981)

26. Kiviloog, $J$.: Bronchial reactivity to exercise and metacholine in bronchial asthma. Scand. J. Respir. Dis., 54: 347 (1973).

27. McFadden, E. R., Jr., Kiser, R. and DeGroot, W. J.: Acute bronchial asthma. Relations between clinical and physiological manifestations. N. Eng. J. Med. 288: 221 (1973).

28. McNicol, K. N. and Williams, H. B.: Spectrum of asthma in children. I. clinical and physiological components. Br. Med. J., 4: 7 (1973).

29. Mellis, C. M., Kattan, M., Keens, T. G. and Levison, H.: Comparative study of histamine and exercise challenge in asthmatic children. Am. Rev. Respir. Dis., 117: 911 (1978).

30. Nadel, J. A. and Tierney, D. F.: Effect of a previous deep inspiration on airway resistance in man. J. Appl. Physiol., 16: 717 (1961).

31. O'Byrne, P. M., Ryan G., Morris, M., McCormack, D., Jones, N. L., Morse J. L. C., and Hargreave, F. E.: Asthma induced by cold air and its relation to nonspecific bronchial responsiveness to metacholine. Am. Rev. Respir. Dis., 125: 281 (1982).

32. O'Cain, C. F., Dowling, N. B., Slutsky, A. S., Hensley, M. J., Strohl, K. P. McFadden, E. R., Jr., and Ingram, R. H., Jr.: Airway effects of respiratory heat loss in normal subjects. J. Appl. Physiol.: Respirat. Environ. Exercise Physiol., 49: 875 (1980).

33. Pavia, D., Thomson, M. L., Clarke, S. W., and Shannon, H. S.: Effect of lung function and mode of inhalation on penetration of aerosol into the human lung. Thorax, 32: 194 (1977)

34. Polgar, G. and Promadhat, V.: Pulmonary Function Testing in Children: Techniques and Standards. (W. B. Saunders, Philadelphia-London-Toronto, 1971).

35. Ryan, G., Dolovich, M. B., Eng, P., Obminski, G., Cockroft, D. W., Juniper, E., Hargreave, F. E., and Newhouse, M. T.: Standardization of inhalation provocation tests: influence of nebulizer output, particle size and method of inhalation. J. Allergy. Clin. Immunol., 67: 156 (1981).

36. Silverman, M. and Anderson, S. D.: Standardization of exercise tests in asthmatic children. Arch. Dis. Child., 47: 882 (1972).

37. Simonssen, B. G., Jacobs, F. M., and Nadel, J. A.: Role of autonomic nervous system and the cough reflex in the increased responsiveness of airways in patients with obstructive airway disease. J. Clin. Invest., 46: 1812 (1967).

38. Solymar, L., Aronsson, P. H., Bake, B., and Bjure, J.: Nitrogen single breath test, flow-volume curves and spirometry in healthy children, $7-18$ years of age. Europ. J. Resp. Dis., 61: 275 (1980).

39. Strauss, R. H., McFadden, E. R., Jr., Ingram, R. H., Jr., and Jaeger, J. J.: Enhancement of exercise-induced asthma by cold air. N. Engl. J. Med., 297: 743 (1977).

40. Strauss, R. H., McFadden, E. R., Jr., Ingram, R. H., Jr., Deal, E. C., Jr., and Jaeger, J. J.: Influence of heat and humidity on the airway obstruction induced by exercise in asthma. J. Clin. Invest., 61: 433 (1978).

41. Swift, D. L.: Generation and respiratory deposition of therapeutic aerosols. Am. Rev. Respir. Dis., 122 (No. 5, part 2): 71 (1980).

42. Taussig, L. M., Chernick, V., Wood, R., Farrell, P., Mellins, R. B., and members of the conference committee: Standardization of lung function testing in children. Proceedings and recommendations of the GAP Conference Committee, Cystic Fibrosis Foundation. J. Pediatr., 97: 668 (1980).

43. Zach, M. S., Schnall, R. P., and Landau, L. I.: Upper and lower airway hyperreactivity in recurrent croup. Am. Rev. Respir. Dis., 121:979 (1980).

44. Zapletal, A., Motoyama, E. K., Gibson, L. E., and Bouhuys, A.: Pulmonary mechanics in asthma and cystic fibrosis. Pediatrics, 48: 64 (1971).

45. Zapletal, A., Samanek, M., and Paul, T.: Upstream and total airway conductance in children and adolescents. Bull. Europ. Physiopathol. Respir., 18: 31 (1982).

46. This work was presented in part in a poster symposium at the Annual Meeting of the American Thoracic Society, Kansas City, May 1983.

47. Visiting professor on sabbatical leave 1982; supported in part by the Bundesministerium für Wissenschaft und Forschung, Austria

48. Requests for reprints should be addressed to: Dozent Dr. Med. Maximilian Zach, Universitäts Kinderklinik, Auenbruggerplatz 30, A-8036 Graz, Austria.

49. Received for publication July 18, 1983.

50. Accepted for publication November 29, 1983.

\title{
Human IgG Antibodies to Carbohydrate and Protein Antigens in Mouse Protection Tests with Group B Streptococci
}

\author{
KAREN K. CHRISTENSEN, ${ }^{(39)}$ POUL CHRISTENSEN, GABRIEL DUC, WALTER H. HITZIG, \\ VIVEKA LINDÉN, BEAT MÜLLER, AND REINHARD A. SEGER \\ Departments of Obstetrics and Gynecology and Medical Microbiology, University Hospital, Lund, Sweden and \\ Department of Pediatrics, Divisions of Hematology-Immunology and Neonatology, University of Zürich,
} Zürich, Switzerland [G.D., W.H.H., B.M., R.A.S.]

\section{Summary}

The protective effect of four commercial human gammaglobulin batches (I-IV) in mice was studied using six different strains of group B streptococci (GBS): types Ia; Ib; II, R-protein negative (R-); II, R+; III, R-; and III, R+. Each mouse received $1.0 \mathrm{ml}$ gammaglobulin and $0.5 \mathrm{ml}$ bacteria, $10^{6}-10^{8}$ colony forming units (CFU). There was a close correlation between antibody levels measured by the use of radiolabeled protein $A$ and the mouseprotective effect of the gamma-globulins. The mouse-protection tests demonstrated that batch I protected against GBS types Ia and III, R- at low concentration (65 mg/ $\mathrm{kg}$ mouse weight), against type Ib at medium ( $260 \mathrm{mg} / \mathrm{kg})$ and against type III, $R+$ at high concentration. Batch IV protected against types Ia and Ib, although the doses were four times higher than those in batch
I, but did not protect against type III, R+. There was no mouse protection by any of the batches against type II.

Antibody levels against Ibc and $\mathbf{R}$, protein antigens, were substantially lower in batch IV. Because the results of these mouse-protection studies indicate the importance of such antibodies against protein antigens, batches I-III might be more useful for therapy of neonatal GBS-septicemia.

\section{Abbreviations}

ELISA, enzyme-linked immunosorbent assay GBS, group B streptococci CFU, colony forming units cpm, counts per minute 